\title{
Application of Chinese herbal medicine additives in aquaculture
}

\author{
Chaolan Guo, Linlin Liang, Ke Cao
}

The Ocean College, Hainan University, Haikou, 570228, China

\begin{abstract}
Keywords: Chinese herbal medicine additive; aquaculture; function; problem; countermeasure
\end{abstract}

\begin{abstract}
Chinese and foreign research achievements prove Chinese herbal medicine additive as an immunopotentiator can well improve immunity of fishes and shrimps, enhance non-specific killing activity of various etiologies and then reduce disease occurrence chance. It opens up a new way for disease control of aquatic products. This paper analyzes basic features of Chinese herbal medicine additive, introduces common Chinese herbal medicine additives and their important functions in aquaculture, sets forth the problems of Chinese herbal medicine additives in aquaculture and proposes corresponding countermeasures.

With continuous economic development in China in recent years, aquaculture industry has gained great development. It is worth noting that disease phenomena of aquatic products become increasingly serious. The varieties and number of fish chemicals used in aquaculture continue to rise. Frequent application of antibiotics and vermifuges give rise to large-scale residue of chemicals in fish bodies, thus leading to pathogen resistance and the decrease in the quality of aquatic products. The health of modern people is jeopardized. Besides, the environment is polluted. Thus, development and production of safe and efficient pollution-free aquaculture chemicals has become the key to better and faster development of aquaculture industry. Chinese herbal medicine additives own numerous advantages such as nature, efficiency, small toxic and side effects and rich resources. They not just have drug property and nutrition in the process of preventing and curing fish diseases, but also can improve production property and high efficiency of feed utilization of aquatic livestock. In view of these, it is urgently necessary to popularize and apply Chinese herbal medicine additives to prevent and cure fish diseases.
\end{abstract}

\section{Basic features of Chinese herbal medicine additives}

Firstly, Chinese herbal medicine additives are wide in resources and low in cost. Since China has a vast territory, Chinese herbal medicine resources are very abundant. It is easy to plan and harvest Chinese herbal medicines. Chinese herbal medicine as a natural substance owns naturality and biological activity of multiple ingredients which can be easily absorbed and utilized. Even if they cannot be absorbed, they can be discharged outside smoothly and discomposed by in-vitro germs, without water environment pollution. However, general chemicals will accumulate in animal bodies for a long term or be left in water. Thirdly, toxic and side effects are small and drug resistance will not form in animal bodies. Components of Chinese herbal medicine and interactions among traditional Chinese medicines can further boost the function (preventing and curing diseases) of Chinese herbal medicine additive so as to weaken or cut down toxic and side effects of drugs. After rational processing and preparing, toxicity of toxic Chinese herbal medicines will decline and even disappear. Meanwhile, medical researches do not find drug resistance of Chinese herbal medicines.

\section{Common Chinese herbal medicine additives and their important functions in aquaculture}

The author through systematically collecting and sorting feels there are many Chinese herbal medicine additives applied in aquaculture, nine of which are mainly introduced as follows. 1) Rheum officinale. The effective ingredient of rheum officinale is anthraquinone derivative, where antibacterial function of rhein, rheum emodin and aloe-emodin are the best. Rheum officinale owns such functions as convergence, increasing blood platelet, promoting blood coagulation and resisting tumor. Besides, it can be used to prevent and cure hemorragic disease of grass carp, bacterial gill-rot 
disease and white head-mouth disease etc. 2) Chinese tallow tree, also called cypress or toxicodendron sylvestre. Its leaves mainly contain alkaloid, flavonoid, tannin and organic acid. Its main antibacterial ingredient is penolic acid and alkali substance which can generate deposit under the function of quick lime and be used to prevent and cure gill rot disease and white head-mouth disease etc. 3) Gllnut. This drug contains tannic acid and has the function of convergence. It can make partial protein of skin mucosa and anabrosis of the body coagulated and promote blood coagulation so as to fulfill hemostasis effect. Gallnut can generate alkaloid and has detoxifying function for body alkaloid. Besides, the antibacterial range is wide. It is an externally applied agent which can be used in bacterial disease of aquatic livestock. 4) Red-knees herb. This drug contains tannin, anthraquinone derivative and polygonum acid etc. It can be mainly used to prevent and cure bacterial enteritis of bodies. 5) Scutellaria baicalensis. It is a kind of perennial vegetation. Its root serves as a medicine and owns such effects as anti-bacteria, anti-virus, calmness, diuresis and detoxication. Besides, it can effectively prevent and treat gill rot disease, sepsis and enteritis. 6) Coptis chinensis. It is a perennial herbaceous plant. Its root stock serves as a medicine and owns such functions as anti-bacteria, antiphlogosis and detoxication. In addition, it can be used to prevent and treat bacillary enteritis. 7) Andrographis herb. It is a annual herb, containing andrographolide and flavonoids etc. and has effects of detoxication, detumescence, pain relief and anti-bacteria. It can be used to prevent and treat bacterial enteritis. 8) Golden cypress which belongs to deciduous trees. Its bark serves as a medicine and owns such functions as anti-bacteria, detoxication, and pain relief etc. it can effectively prevent and treat hemorragic disease of grass carps. 9) Garlic which belongs to officinal bulbs. Its effective ingredient is allicin. It owns such important functions as anti-diarrhea, sterilization and parasite expelling and can be used to prevent and treat bacterial intestinal diseases.

Chinese herbal medicine additives have two important functions in aquaculture. Firstly, they have immunologic enhancement function. Immunologic function of Chinese herbal medicines is reflected in stimulating non-specific immunity. For example, milk vetch and scutellaria baicalensis are mixed with feeds to feed 3-mongth-old tilapia mossambica for 4 weeks. The results prove that the above two herbal medicines can activates phagocytic activity of leukocyte and serum lysozyme activity in blood. Meanwhile, milk vetch can boost phagocytic activity of leukocyte in the blood of Chinese softshell turtles, while fresh ginger extractive can significantly promote phagocyte activity in the blood of rainbow trout. For another example, Chinese herbal medicines are added in prawn feeds, which can activate nonspecific immunity of prawns and improve hemagglutinin activity and bacteriolysis activity. It can be found from the above researches that effects of Chinese herbal medicines on nonspecific immunity are mainly reflected in lysozyme activity owned by Chinese herbal medicine additive and phagocytic activity owned by phagocyte. Secondly, they have the function of preventing and treating diseases. The cost of Chinese herbal medicine additives is low and the curative effect is ideal. Besides, they are green and environmentally friendly. So, they have been widely used in preventing and treating diseases of aquatic livestock. In terms of preventing and curing virosis, hemorragic disease of grass carps is the most common. Chinese herbal medicines can be used to prevent and treat hemorragic disease of grass carps include rheum officinale, golden cypress, folium isatidis and verbena etc. Experiments prove that rheum officinale and algae iodate also have quite good curative effect for hemorragic disease of grass carps. The survival rate can increase to $17 \%$ - 30\%. Of course, Chinese herbal medicine additives also have good effects for bacteriosis. For instance, researchers use rheum officinale, scutellaria baicalensis, golden cypress, coptis chinensis and radix lsatidis to prevent and treat streptococcicosis of rainbow trout. The curative effect is quite significant, compared with the control group. In the third day of medicine adding, the number of dead fish reduced obviously. Death stops in the 7th day. The fish basically returns to normal. For another instance, rheum officinale, golden cypress, scutellaria baicalensis, the root of Chinese pulsatilla, folium artemisiae argyi and fructus forsythiae have anti-bacterial effect for monad which triggering gill rot disease and bacillary enteritis of fishes. Moreover, skin mildew of fishes is mainly caused by water molds and pythium which grow on the surface of fish bodies. It is one of fish diseases with serious harm. Pericarpium citri reticulatae and 
folium artemisiae argyi possess antifungal activity. Cinnamaldehyde and anisaldehyde extracted from Chinese herbal medicines own quite strong anti-mould effects.

\section{Problems of Chinese herbal medicine additives in aquaculture}

Firstly, fundamental researches on application of Chinese herbal medicine additives in aquaculture lag behind in Chins. Currently, regardless of single preparation or compound preparation, the functions of Chinese herbal medicines used in aquaculture mainly refer to historical books and records about traditional Chinese medicine and experience in clinical medication. However, Chinese herbal medicine theories in traditional sense are badly short of analysis of effective ingredients of Chinese herbal medicine and researches on mechanism of antivirus activity of Chinese herbal medicines. Drug sensitive test and dissection experiment will not be conducted like western medicine. So, clinical reactions and clinical experiment data are very scarce. If experimental researches are carried out one by one from perspective of pharmacology, there is the lack of relevant technical researches and corresponding funds. Therefore, during applying Chinese herbal medicine additives, incompatibility problem should be highly valued. Secondly, the study and development of Chinese herbal medicine additives receive more and more attention, but industrial base is weak and capital input seriously lags behind. Although researches on immunopotentiator and especially immunopotentiator feed additives have been on the rise in recent years, fundamental study and development of Chinese herbal medicines are still quite poor on the whole. The manufacturing technique falls behind extremely, and engineering level is low. Chinese herbal medicine enterprises generally have small scale, but the number of enterprises is large. Besides, protect repetition phenomenon is quite serious. They have low technological content, low management level and low automatic production level. Meanwhile, dosage form of Chinese herbal medicine also lags behind. The funds in studying Chinese herbal medicines are so few that the researches on Chinese herbal medicine additives applied in aquaculture industry cannot adapt actual needs. Thirdly, dosage form of Chinese herbal medicine additives is very chaotic. There are great obstacles in digestion and absorption, which thus seriously affects medicine functions. Currently, Chinese herbal medicine additives applied to prevent and treat diseases of aquatic products have diversified dosage forms, mainly including powder and aqueous solution. Powder medicines include common smashed agent and superfine ground agent, while aqueous solution includes aqueous solution decocted with water, aqueous solution extracted from chemicals and supercritical extraction with carbon dioxide. Most dosage forms exist in the form of common powder. Ingredients of most Chinese herbal medicines are of crude fiber and chitinous substance. Special digestion structure of aquatic livestock decides very poor digestive absorption effects for crude fiber and chitinous substance. Thus, the effects of common powder are not very ideal due to digestion and absorption obstacles caused to aquatic livestock.

\section{Countermeasures to apply Chinese herbal medicine additives in aquaculture}

Firstly, intensify basic theoretical researches on Chinese herbal medicine additives. At present, the researches on Chinese herbal medicines applied in aquaculture concentrate on clinical application and effective ingredients. Effective ingredients, content, structure, extraction and mutual relations among effective ingredients, toxicology and pharmacology of a large number of Chinese herbal medicines and compound preparations lack such functions as growth promotion, disease prevention and treatment of aquatic animals as well as aquatic product quality improvement. It is required to deeply study features and functions of Chinese herbal medicine additives to screen out Chinese herbal medicines with good effects. Secondly, establish hand perfect Chinese herbal medicine research system oriented to market disciplines. Food source pesticides and residues of veterinary drugs seriously endanger health of modern people, while toxic and side effects and drug tolerance of chemicals and antibiotics become increasingly outstanding. This cusses people have to transfer prevention and treatment of diseases and especially animal diseases to new objective of Chinese herbal medicine researches. In view of this, the state starts to support a batch of large 
enterprise which own proprietary intellectual property rights and are rich in market competitiveness and set up new-type Chinese herbal medicine additive industry which contributes to overall economic development and regional economic development and own strong competitive advantage in market. Thirdly, closely combine the digestion and absorption principle of aquatic animals and mainly change dosage forms of Chinese herbal medicine additives in aquaculture. For example, superfine grinding or supercritical extraction with carbon dioxide can be used. In this way, digestion and utilization efficiency of Chinese herbal medicine additives can greatly improve, and then drug effects will also increase. Fourthly, uniform quality standards should be used. The quality and producing area of Chinese herbal medicine additives should be strictly controlled. Meanwhile, raw material pollution should be avoided as far as possible so as to make the prescription become more rational and scientific. Fifthly, rational combination of Chinese herbal medicine additives and western medicine can practically make up for shortcomings of slow effects of Chinese herbal medicines so that prescription medicine can be faster and better.

\section{Conclusions}

In conclusion, as current society develops to environmental protection and healthy direction, aquaculture is also of no exception. Nowadays, various countries are continuously increasing input in scientific researches and positively seeking new channels of green cultivation. One of impotent researches is to develop low-pollution, low-cost and wide-distribution Chinese herbal medicine additives with various purposes to replace original antibiotics. Chinese herbal medicines are every rich in reserve, and the application history is very long. So, they with good application value can be easily accepted by social public. But, the application of Chinese herbal medicine additives in aquaculture also has significant problems. So, development tendency of Chinese herbal medicines should be as follows: strive to achieve standard production, enhance detection of biological activity of the extract, specify effective ingredients, pharmacology and principles of pharmacokinetics and form standard quality monitoring system such as extraction technology and production flow. In this way, superior and effective Chinese herbal medicine products can be really produced. The author firmly believes that through mutual combination of Chinese medicine technology and modern high-tech means, the development of Chinese herbal medicine additives will certainly step to a new stage and bring larger benefits for Chinese aquaculture industry.

\section{References:}

[1] Chen Gang, Features Chinese herbal medicine additives and their application in aquaculture [J]. Technical Advisor for Animal Husbandry, 2012 (12)

[2] Song Shiming, Application of Chinese herbal medicine additives in aquaculture [J]. Journal of Rural Animal-Breeding Technology, 2013 (6)

[3] Chen Liting, Research progress of application of Chinese herbal medicine additives in aquaculture [J]. Fisheries Science, 2014 (3)

[4] Huang Huafeng, Methods to apply Chinese herbal medicine additives in aquaculture [J]. South China Agriculture, 2014 (9)

[5] Du Qiang, Action mechanism and application prospect of Chinese herbal medicine additives in aquaculture [J]. Feed Research, 2014 (11) 